\title{
IS THE PLEOTROPIC EFFECT OF RANOLAZINE IS DUE TO ITS ANTIOXIDANT ACTION
}

\author{
Janapati Rama Krishna, Vijay Kumar, Narendra Kumar, Garre Indrani
}

\begin{abstract}
BACKGROUND: Ranolazine is indicated as antianginal drug. We observed that it has other pleotropic effects also. So, we want to study the effect of Ranolazine in improvement of left ventricular (LV) function in obstructive coronary artery (CAD) diseased patients along with ACC/AHA guidelines of treatment. And to see the mode of improvement in LV function is due to its antioxidant effect.

MATERIALS AND METHODS: We recruited CAD patient between the age group 18-80 yrs with LV dysfunction in whom ischemic component was treated. Cases received ranolazine $500 \mathrm{mg}$ two times a day and controls without the drug. Both groups received standard drug therapy according to ACC/AHA guidelines of treatment CAD. For all these patients at basal and at 6 months of follow up, all baseline and demographic parameters, clinical features and symptomatology with blood chemistry parameters were collected. In addition Two Dimensional echocardiography (EF, MAV), Treadmill test (METs) and plasma oxidative stress levels (MDA) will be performed at 0 day (day of recruitment) and at 6 months. MDA concentration was tested by using the method described by Draper and Hadley based on TBA reactivity. Normal Range of MDA is $3.60 \pm 0.90$ Nano mole/ml .

RESULTS: 100 cases and 40 controls were recruited for this study. Male: Female ratio was 3.4:1 in cases and in controls. The mean age of cases was $56.8 \pm 9.6 \mathrm{yrs}$ in cases and $55.1 \pm 12$ yrs in controls. There was no difference in demographic features and risk factor profile in between the cases and controls expect smoking ( high in cases). Even in echo EF was comparable but MAV is more in cases than controls (9.5 \pm 2.1 vs $8.8 \pm 1.8$ ) which are also not statistically significant.

There is statistically significant improvement of EF either by dimension $(48.3 \pm 9.6$ vs $43.8 \pm 8.4 \%, p=0.008)$ or volume $(p=0.018)$ method of estimation and peak mitral annular velocity in cases $(9.8 \pm 1,8$ vs $8.7 \pm 1.8 \mathrm{~cm} / \mathrm{sec}, \quad p=0.001)$ who received additional ranolazine therapy than controls.
\end{abstract}

Article received on 25 DEC 2015, published on 10 JAN 2016.

Rama Krishna ${ }^{1}$, Vijay Kumar ${ }^{2}$, Narendra Kumar ${ }^{3}$, Indrani Garre ${ }^{4}$,

${ }^{1}$ Senior resident, Departments of Cardiology, NIMS, India

${ }^{2}$ Additional Professor, Department of Clinical Pharmacology \& Therapeutics

${ }^{3}$ Cardiovascular technologist, Departments of Cardiology

${ }^{4}$ Clinical study coordinator, Departments of Cardiology

Corresponding author: Rama Krishna

Email: ramakrishnajanapati@gmail.com
MDA at 6 months in cases was decreases to $3.4 \pm 0.8$ nano mole/dl where as in controls increased to $3.9 \pm 0.7$ nano mole/dl. Out of 100 cases we could able to get Treadmill test done only for 89 patients with modified Bruce protocol and there was a statistically significant improvement in the number of metabolic equivalents at 6 months in cases comparison to basal exercise capacity (6.8 \pm 2.4 vs $8.2 \pm 8.1$ METS, $p=0.05)$.

Conclusions

Ranolazine can act as not only an anti anginal drug but also it was shown to have positive effects on improving LV function (improvement in EF and mitral annular velocities) and improving the exercise capacity (increased METs by TMT) . These effects may be due to reduction in the oxidative stress (decreased MDA levels).

Key words: Ranolazine. MDA, EF.

\section{INTRODUCTION}

Ranolazine a Piperazine derivative is an unique anti anginal drug. The mechanism of action of this agent remains under investigation. When studied at high concentrations in invitro experiments, Ranolazine was shown to shift myocardial substrate utilization from fatty acid to glucose and thus was considered to be a potential metabolic agent. Apart from its anti anginal action it was shown to have pleotropic effects and also likely anti-arrhythmic action due to its effects on after repolarizations. In previous studies it was proven that inhibition of late I Na currents may attenuate reactive oxygen species-induced cardiac dysfunction. Oxidative stress reflects an imbalance between the systemic manifestation of reactive oxygen species and a biological system's ability to detoxify the reactive intermediates or to repair the resulting damage. We tested the oxidative stress marker levels in ischemic LV dysfunction patients to see where the positive LV remodeling is due to anti-oxidant effect of the Ranolazine.

AIM: In this study we aimed to study the role of Ranolazine in improving the LV function by various echocardiography parameters and exercise capacity and its role in reducing the levels of a novel oxidative marker Malonaldehyde. And to study the feasibility of 
using Ranolazine for left ventricular remodeling in PCI treated patients with LV dysfunction.

\section{METHODS:}

Study population: One hundred (100) cases and forty controls (40) with LV dysfunction were recruited in this study with 6 months of follow up and all baseline and demographic parameters, clinical features and symptomatology with blood chemistry parameters were collected for all patients.

Inclusion criteria: Patients (age group 18-80 yrs) with coronary artery disease with LV dysfunction in which ischemic component was treated.

Exclusion criteria: Patients with untreated coronary ischemia, comorbidities which effects the survival of patients such as advanced malignancy, chronic renal and liver failure. Patients who has contraindications to long and short term antiplatelet or anticoagulant therapy such as bleeding disorders, gastrointestinal or intracranial bleeding, recent cerebrovascular accident, recent major surgery were excluded.

Both groups will receive standard drug therapy according to ACC/AHA guidelines of treatment coronary artery disease. In addition cases will receive ranolazine $500 \mathrm{mg}$ two times a day. Two Dimensional echocardiography, Treadmill test and plasma MDA will be performed at 0 day (day of recruitment) and at 6 months.

2D ECHOCARDIOGRAPHY: Sonus 5000 echocardiographic machine was used in this study. The parameters studied were ejection fraction [ $\{\mathrm{LV}$ volume in end diastole (LVEDV) - LV volume in end systole (LVESV)\}/ LVEDV\}, fractional shortening ((LVEDdLVESd) / LVEDd expressed as a percentage - $d$ is dimension) and peak mitral annular velocity (degree of movement of mitral annulus during systole - PMAV) by Tissue Doppler. PMAV was recorded with $4 \mathrm{MHz}$ probe as in previous studies which were more reliable in wide variety of coronary artery diseases patients with regional ischemia, different shapes and sizes of LV; we selected this parameter for assessment of LV contractility. Normal peak MAV $>8 \mathrm{~cm} / \mathrm{sec}$.
By 2D and Doppler echocardiogram we collected the data of LV end systolic dimension(ESD), end diastolic dimension (EDD), EF by dimensions \{(EDD3ESD3)/ESD3\}, End diastolic volume (EDV), end systolic volume (ESV) EF by Simpson's method \{(EDV$\mathrm{ESV}) / \mathrm{EDV}\}$, mitral inflow and pulmonary velocities both pulse wave and tissue Doppler (E, A, E/A, e', a', e'/a', PV $\mathrm{s}^{\prime}, \mathrm{PV} \mathrm{a}^{\prime}$ and duration).

OXIDATIVE STRESS LEVELS: Various Oxidative stress indicators are plasma malondialdehyde (MDA) \& protein carbonyl (PC) content and protective antioxidant sulfhydryl content. Malondialdehyde (MDA) is one of the most frequently used indicators of lipid peroxidation. We used plasma MDA levels to know the degree of oxidative stress. MDA concentration will be determined by using the method described by Draper and Hadley based on TBA reactivity. According to this method, $2.5 \mathrm{~mL}$ of $10 \%$ trichloracetic acid and $0.5 \mathrm{~mL}$ of plasma will be added into tubes and mixed. After incubating for $15 \mathrm{~min}$ at $90^{\circ} \mathrm{C}$ and cooling with cold water the mixture will be centrifuged at $3000 \mathrm{rpm}$ for 10 min. Two milliliters of supernatant will be taken and $1 \mathrm{ml}$ of $0.675 \%$ TBA will be added. The tubes will be sealed and incubated at $90^{\circ} \mathrm{C}$ for $15 \mathrm{~min}$ and then cooled to room temperature. The optical density will be measured at $532 \mathrm{~nm}$ by a spectrophotometer. Normal Range of MDA is $3.60 \pm 0.90$ nano mole $/ \mathrm{ml}$.

STATISTICS: Minitab 16 version is used for statistical analysis. For continuos variables chi-square test used and for categorical variables we used $t$ test (pvalue $<0.005$ ) Analysis of variance was performed to check whether there is any significant difference between the basal values, 6 months control \& with drug $(\mathrm{F}=5.8, \mathrm{p}=0.001)$. We performed further analysis of these three important echo parameters by calculating capability indices

\section{RESULTS}

100 cases and 40 controls were recruited for this study. Male: Female ratio was 3.4:1 in cases and in controls. The mean age of cases were $56.8 \pm 9.6$ yrs in cases and $55.1 \pm$ 12 yrs in controls. (Fig 1). 
Fig 1: Age distribution in controls and cases.

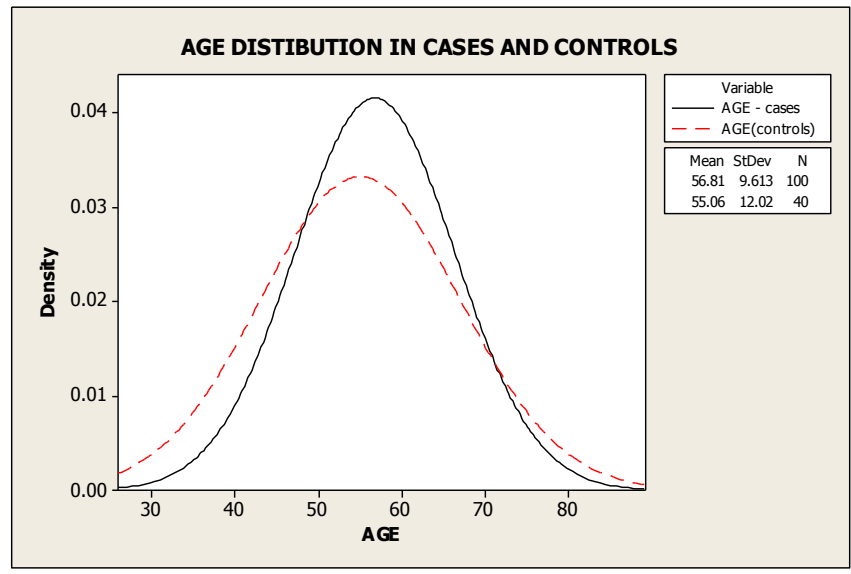

The details of average height, weight, hemoglobin $(\mathrm{Hb})$, packed cell volume (PCV), total leukocyte count (TLC), platelet count (PLT), systolic blood pressure (S BP), diastolic blood pressure (DBP) and plasma Malonaldehyde (MDA) levels of cases and controls were mentioned in Table 1 and 2 respectively.

Table 1 : Clinical and demographic features in cases.

\begin{tabular}{|c|c|c|c|c|}
\hline Variable & Mean & StDev & $\begin{array}{l}\text { Mini } \\
\text { mum }\end{array}$ & $\begin{array}{l}\text { Maxi } \\
\text { mum }\end{array}$ \\
\hline Weight(KGs) & 158.82 & 22.36 & 60 & $\begin{array}{l}201.6 \\
7\end{array}$ \\
\hline Hight(cms) & 60.97 & 19.25 & 26.35 & 152 \\
\hline $\mathrm{SBP}(\mathrm{mm} \mathrm{Hg})$ & 125.5 & 10.4 & 115 & 160 \\
\hline $\mathrm{DBP}(\mathrm{mmHg})$ & 81.3 & 10.8 & 72 & 98 \\
\hline $\mathrm{Hb}(\mathrm{g} / \mathrm{dl})$ & 13.1 & 1.242 & 10.5 & 15.7 \\
\hline PCV $(\mu \mathrm{l})$ & 39.145 & 4.176 & 29.9 & 48.1 \\
\hline $\begin{array}{l}\text { TLC } \\
\text { (cell/cu.mm) }\end{array}$ & 8213 & 1457 & 4800 & 11500 \\
\hline PLT (lakh/dl) & 2.685 & $\begin{array}{l}0.493 \\
3\end{array}$ & 1.7 & 3.7 \\
\hline $\begin{array}{l}\mathrm{MDA}(\mathrm{nmol} / \mathrm{m} \\
\text { l) }\end{array}$ & 3.8 & 1.1 & & \\
\hline
\end{tabular}

For comparison of presence of risk factors (attributable data) like hypertension (HTN), diabetes mellitus (DM) and smoking (SM) in cases and controls were done with two proportion test Table 3. Basal clinical, demographic features and risk factors are comparable between cases and controls except for smoking which was more frequent in cases than controls.
Table 2: Clinical and demographic features in controls

\begin{tabular}{|l|llll|}
\hline Variable & Mean & StDev & Min & Max \\
\hline Weight(Kgs) & 158.82 & 22.36 & 60 & 201.67 \\
\hline Height(cms) & 60.97 & 19.25 & 26.35 & 152 \\
\hline SBP(mm Hg) & 125.5 & 10.4 & 115 & 160 \\
\hline DBP((mm Hg) & 81.3 & 10.8 & 72 & 98 \\
\hline Hb(g/dl) & 13.1 & 1.242 & 10.5 & 15.7 \\
\hline PCV $(\mu l)$ & 39.145 & 4.176 & 29.9 & 48.1 \\
\hline TLC(cell/cu.mm) & 8213 & 1457 & 4800 & 11500 \\
\hline PL (lakh/dl) & 2.685 & 0.4933 & 1.7 & 3.7 \\
\hline MDA(nmol/ml) & 3.8 & 1.1 & & \\
\hline
\end{tabular}

We compared the clinical and lab variables which influence the LV remodeling such age $\mathrm{Hb}$, systolic and diastolic blood pressures and MDA levels in cases and controls at the time of recruitment, which are comparable (Table 4).

With 2D echocardiography we noted the base line EF (by dimension and by volume) and peak MAV in both groups (cases and controls). The scatter diagram of EF estimation by volume and dimensions shows that these is good correlation between these two methods so that either of it we may use for LV remodeling. Important echocardiographic variables such as EF and MAV are comparable in cases and controls at the time of recruitment (Table 5).

We tested the distribution of MAV, MDA and EF. MAV and MDA are not following the normal distribution. Both the parameter are following Lognormal $(p=0.436)$,Gamma $(p=0.209)$,Loglogistic $(p=0.216)$,BoxCox Transformation $(\mathrm{p}=0.522)$ and Johnson Transformation $(\mathrm{p}=0.99815)$ distributions. Whereas EF by volume estimation both in controls and cases is following normal distribution.

Out of 100 cases we could able to get Treadmill test done only for 89 patients with modified Bruce protocol and there was a statistically significant improvement in the number of metabolic equivalents at 6 months in cases comparison to basal exercise capacity (Table 6) 
Table 3: Comparison of demographic and risk factors in cases and controls at time of recruitment of study (Basal).

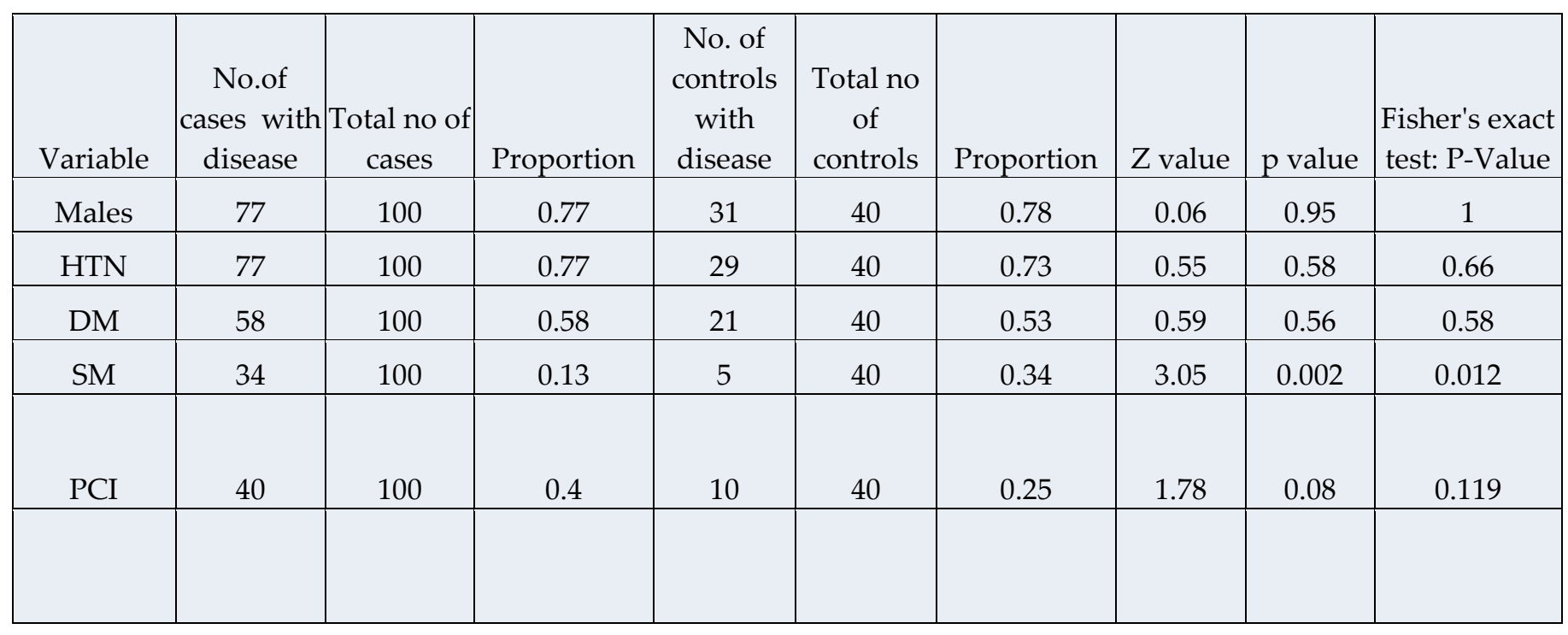

Table 4 : Comparison of Basal variables which influence the LV remodeling in cases and controls

\begin{tabular}{|l|l|l|l|l|l|l|}
\hline Variables & Cases (mean) & Controls (mean) & Cases (STD) & $\begin{array}{l}\text { Controls } \\
(\mathrm{STD})\end{array}$ & T-value & p-value \\
\hline Age $(\mathrm{yr})$ & 56.8 & 55.1 & 9.6 & 12 & 0.88 & 0.38 \\
\hline $\mathrm{Hb}(\mathrm{g} / \mathrm{dl})$ & 12.9 & 13.1 & 1.3 & 1.2 & 0.84 & 0.4 \\
\hline $\mathrm{S} \mathrm{BP}(\mathrm{mm} \mathrm{HG})$ & 127.4 & 125.5 & 12.4 & 10.4 & 0.92 & 0.36 \\
\hline $\mathrm{D} \mathrm{BP}(\mathrm{mm} \mathrm{HG})$ & 82.2 & 81.3 & 8.3 & 10.8 & 0.47 & 0.64 \\
\hline $\mathrm{MDA}(\mathrm{nmol} / \mathrm{ml})$ & 3.6 & 3.8 & 1.2 & 1.1 & 0.91 & 0.36 \\
\hline
\end{tabular}

Table 5: Basal Echocardiographic parameters in Cases and Controls.

\begin{tabular}{|l|l|l|l|l|l|l|}
\hline Echo variables & $\begin{array}{l}\text { Cases } \\
(\text { mean })\end{array}$ & $\begin{array}{l}\text { Cases } \\
(\text { STD })\end{array}$ & $\begin{array}{l}\text { Control } \\
(\text { mean })\end{array}$ & $\begin{array}{l}\text { Control } \\
(\text { STD })\end{array}$ & T value & p value \\
\hline EF (diameter -\%) & 46.1 & 10.5 & 44.2 & 10.8 & 0.98 & 0.33 \\
\hline EF (volume - \%) & 43.8 & 9.3 & 41.8 & 9.52 & 1.14 & 0.26 \\
\hline MAV $(\mathrm{cm} / \mathrm{s})$ & 9.5 & 2.1 & 8.8 & 1.8 & 1.92 & 0.06 \\
\hline
\end{tabular}

Table 6 : Difference in METs by TMT before after treatment with Ranolazine at 6 months.

\begin{tabular}{|l|l|l|l|l|l|}
\hline & $\mathrm{N}$ & Mean & St Dev & SE mean & $\begin{array}{l}\text { p value } \\
\text { (t value) }\end{array}$ \\
\cline { 1 - 5 } Basal mets & 89 & 6.755 & 2.457 & 0.260 & \multirow{2}{*}{0.05} \\
\cline { 1 - 4 } Mets at 6 month & 89 & 8.273 & 8.069 & 0.855 & $(1.94)$ \\
\cline { 1 - 3 } $\begin{array}{l}\text { Improvement in } \\
\text { mets }\end{array}$ & 89 & 1.518 & & & \\
\hline
\end{tabular}


Table 7: At 6 months Echocardiographic parameters in Cases and Controls

\begin{tabular}{|l|l|l|l|l|l|l|}
\hline Echo variables & $\begin{array}{l}\text { Cases } \\
(\text { mean })\end{array}$ & $\begin{array}{l}\text { Cases } \\
(\mathrm{STD})\end{array}$ & $\begin{array}{l}\text { Control } \\
(\text { mean })\end{array}$ & $\begin{array}{l}\text { Control } \\
(\mathrm{STD})\end{array}$ & T value & p value \\
\hline EF (diameter $-\%)$ & 48.32 & 9.63 & 43.85 & 8.38 & 2.73 & 0.008 \\
\hline EF (volume $-\%)$ & 46.72 & 9.34 & 42.55 & 9.1 & 2.43 & 0.018 \\
\hline MAV $(\mathrm{cm} / \mathrm{sec})$ & 9.79 & 1.84 & 8.65 & 1.81 & 3.36 & 0.001 \\
\hline
\end{tabular}

Fig 2: IMR chart of MDA combining both cases and controls

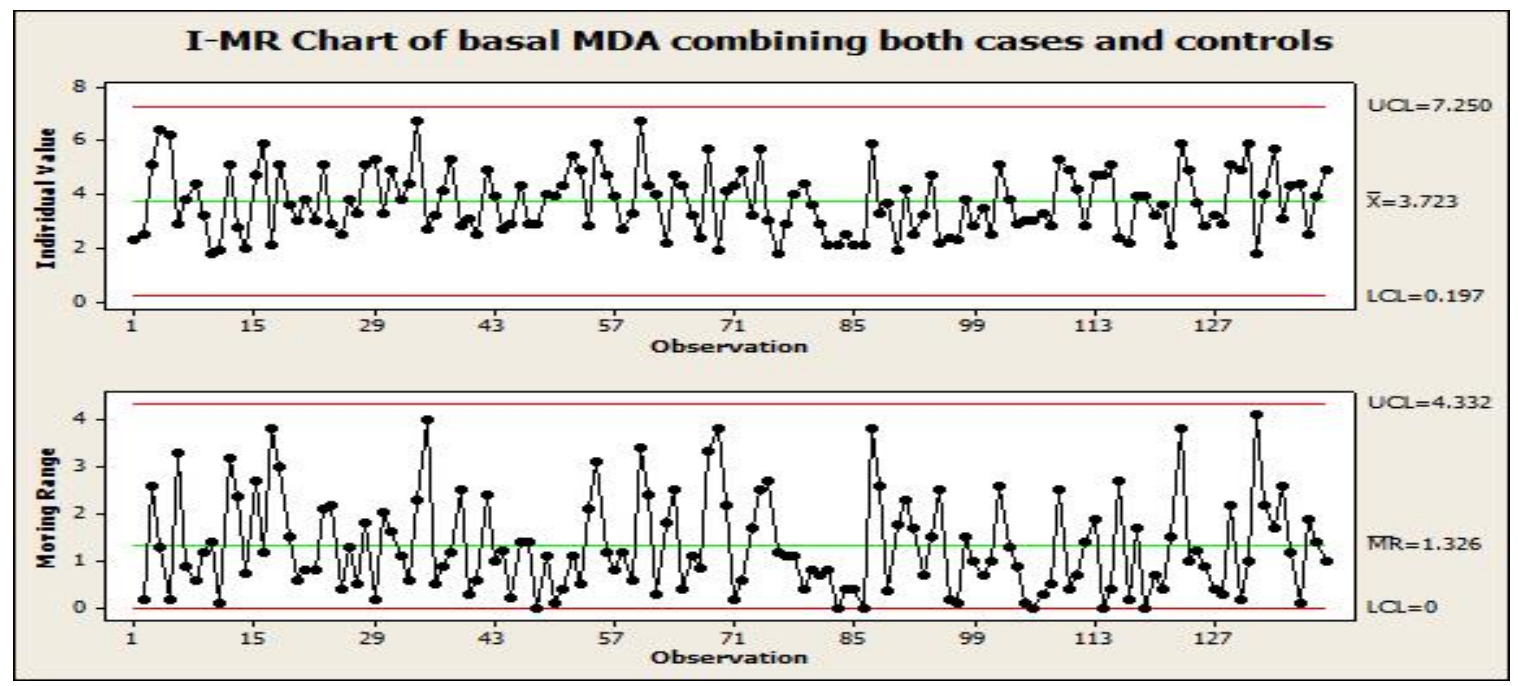

Fig 3 : Comparison of EF in cases and controls at 6 months.

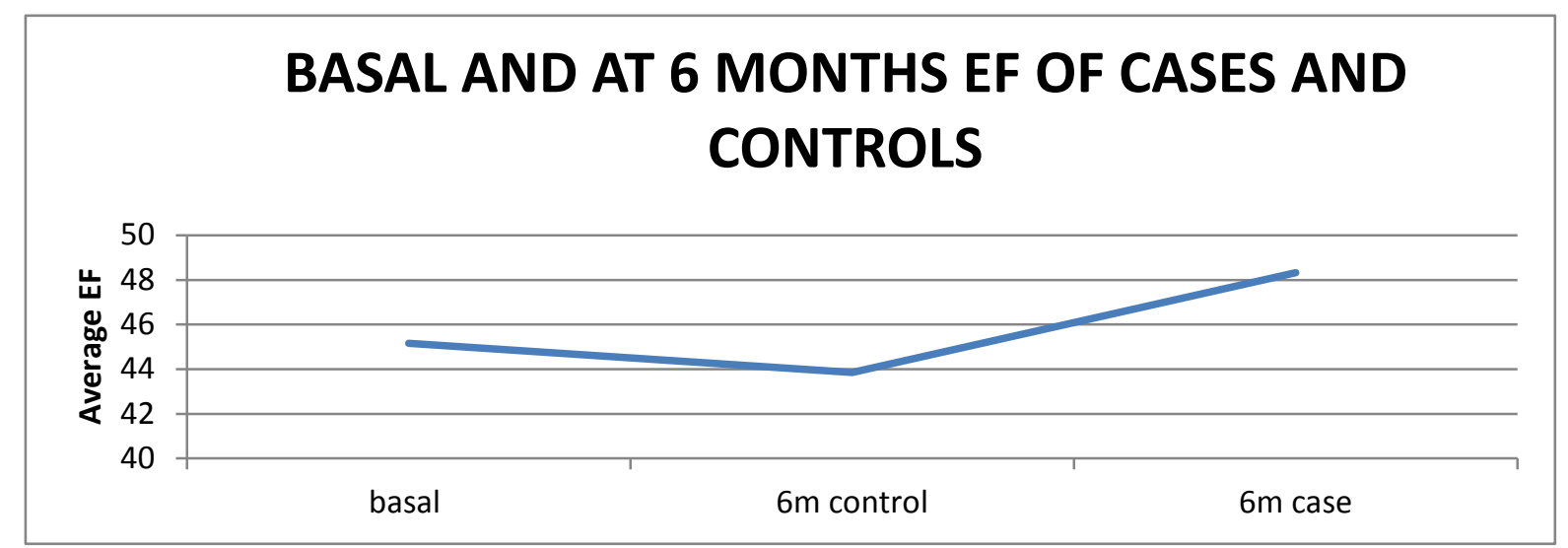

We tested the stability of plasma MDA level estimation with I-MR chart by combining the plasma MDA levels of both cases and controls, which showed proper control of the process (Fig 6). So, we accepted this methodology for estimating subsequent 6 month plasma levels of MDA. After 6 months of drug therapy with ranolazine in addition to standard treatment in cases and in controls without ranolazine only with standard therapy plasma
MDA and different echocardiographic parameters were noted (Table 7). There is statistically significant improvement of EF either by dimension or volume method of estimation and peak mitral annular velocity in cases who received additional ranolazine therapy than controls. 
Table 8: Comparison of MDA at basal and at 6 months in cases and controls

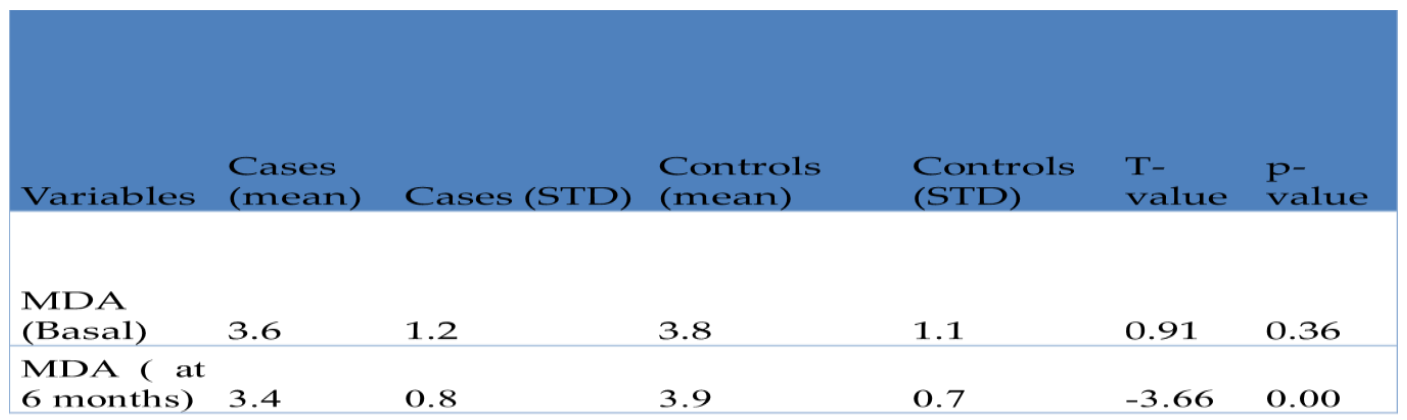

There is significant difference between basal/control and case. The above response plot shows deterioration of EF in controls were as in cases there is improvement. Plasma MDA levels also showed improvement in cases than in controls.

MDA at 6 months in cases was decreases to $3.4 \pm 0.8$ nano mole/dl where as in controls increased to $3.9 \pm 0.7$ nano mole/dl. (Table 8).
Analysis of variance was performed to check whether there is any significant difference between the basal values, 6 months control \& with drug $(\mathrm{F}=5.8, \mathrm{p}=0.001)$. We performed further analysis of these three important echo parameters by calculating capability indices (Table 9).

Table 9: comparison of MVA in cases and controls at basal and 6 months

\begin{tabular}{|l|l|l|l|l|l|l|l|l|l|l|l|}
\hline Variable & Count & Mean & SE Mean & StDev & Min & Q1 & Median & Q3 & Max & Cpk & Threat \% \\
\hline Control (Basal) & 40 & 8.775 & 0.284 & 1.797 & 6.5 & 7.45 & 8.4 & 9.675 & 15 & 0.14 & 34.1 \\
\hline Control (6 months) & 40 & 8.647 & 0.286 & 1.806 & 6 & 7.025 & 8.1 & 9.925 & 14.5 & 0.12 & 36.0 \\
\hline Study (Basal) & 100 & 9.554 & 0.216 & 2.162 & 5.3 & 7.8 & 9.2 & 10.7 & 15.7 & 0.24 & 23.6 \\
\hline Study (6 months) & 100 & 9.789 & 0.184 & 1.839 & 5.3 & 8.525 & 9.7 & 11 & 14.3 & 0.32 & 16.5 \\
\hline
\end{tabular}

It is evident from the ANOVA analysis as well as the capability analysis the cardiac health has significantly improved on use of the drug. The risk has significantly reduced to $16.5 \%$ (estimated \% of patients above 8 , which is considered as high risk). It can be inferred from the study that the effect of Ranolazine on left ventricular remodeling in obstructive coronary artery disease patients is highly significant and has the potential to reduce the risk considerably.

\section{DISCUSSION}

This study demonstrates that treatment with Ranolazine prevents progressive LV dysfunction and attenuates LV remodeling in patients with LV dysfunction. In the present case control study, we examined the effects of treatment with Ranolazine on improvement in LV function from baseline, and improvement in exercise capacity and reduction in oxidative stress levels at 6 moths of follow up. Indices of global ventricular function, such as ejection fraction, were better in the ranolazine group compared with the control group at 6 months. In a study by Vincent M. Figueredo[1], Global left ventricular function, as assessed by the myocardial performance index, was significantly improved on drug therapy $(\mathrm{P}<.0001)$. This was due to improvement in both diastolic and systolic parameters. Similarly our study patients also showed an improvement in LV function as assessed by ejection fraction. Peak Mitral velocities[2] are exhibiting stable (normal) behavior with respect to all the 4 conditions (control \& study). So, this echo parameter can be used to follow the cases in clinical practice to know the improvement in LV contractility like standard EF. In an animal model of heart failure by Sharad Rastogi et al[3] Ranolazine prevented progressive LV dysfunction and global and cellular 
myocardial remodeling, and Ranolazine in combination with Enalapril or Metoprolol improved LV function beyond that observed with Ranolazine alone. Our study was conducted in patients with ischemic heart disease and those who underwent PCI and persisting LV dysfunction. In a study published by Tocchetti CG[4] et al in animal experiments it was shown that Ranolazine protects against experimental doxorubicin cardio toxicity. Such protection is accompanied by a reduction in oxidative stress[5,6] suggesting that INa modulates cardiac redox[7] balance. In our study we used malonaldehyde[8] as on oxidative stress maker which was shown to decreased from baseline in cases in comparison to controls. Hale SL[9] et al studied ranolazine's effect on left ventricular function and infarct size in the setting of acute ischemia, anesthetized rabbits were pretreated with either ranolazine infusion prior to coronary artery occlusion and reperfusion. Rabbits treated with ranolazine had better global left ventricular ejection fraction $(0.42 \%$ vs $0.33 \% ; \mathrm{P}=$ $0.007)$,stroke volume $(1.05 \mathrm{~mL}$ vs $0.78 \mathrm{~mL} ; \mathrm{P}=0.01)$ and smaller infarct size ( $44 \%$ vs $57 \%$; $\mathrm{P}=0.04)$. These findings argue that ranolazine may improve left ventricular systolic function and provide myocardial protection during an acute myocardial infarction.

Ranolazine by inhibiting the late I $\mathrm{Na}$ and preventing pathologic intracellular calcium accumulation in heart failure and ischemic conditions, may be useful in preventing the abnormal myocardial remodeling that leads to systolic and diastolic heart failure[10]. Our study was a first of this kind in humans to demonstrate objective evidence of improved left ventricular systolic function by various echocardiographic methods and decreased novel oxidative stress marker from baseline Conclusions

Peak Mitral velocities can be used to follow the cases in clinical practice to know the improvement in LV contractility like standard EF. There is significant improvement in EF in cases when compared to controls at 6 months, reconfirming the results from the PMAV analysis. There is reduction in oxidative stress levels in cases than controls at 6 months, may be possible mechanism of action of ranolazine induced positive LV remodeling.

Ranolazine can act as not only an anti anginal drug but also it was shown to have positive effects on improving LV function and improving the exercise capacity and these effects may be due to reduction in the oxidative stress.

\section{REFERENCES:}

1. Vincent M. Figueredo, Gregg S. Pressman, Abel Romero-Corral et al. Improvement in Left Ventricular Systolic and Diastolic Performance During Ranolazine Treatment in Patients With Stable Angina J CARDIOVASC PHARMACOL THER June 1, 2011 16: 168-172.

2. Seo JS, Kim DH, Kim WJ et al. Peak systolic velocity of mitral annular longitudinal movement measured by pulsed tissue Doppler imaging as an index of global left ventricular contractility Am J Physiol Heart Circ Physiol. 2010 May;298(5):H1608-15.

3. Sharad Rastogi, Victor G. Sharov, Sudhish Mishra et al. Ranolazine combined with enalapril or metoprolol prevents progressive LV dysfunction and remodeling in dogs with moderate heart failure. American Journal of Physiology 1 November 2008Vol. 295no. H2149H2155.

4. Tocchetti CG , Carpi A, Coppola C et al. Ranolazine protects from doxorubicin-induced oxidative stress and cardiac dysfunction. Eur J Heart Fail. 2014 Jan 6. doi: 10.1002.

5. Song Y, Shryock JC, Wagner S, et al. Blocking late sodium current reduces hydrogen peroxideinduced arrhythmogenic activity and contractile dysfunction J Pharmacol Exp Ther. 2006 Jul;318(1):214-22. Epub 2006 Mar 24.

6. Aldakkak M , Camara AK, Heisner JS,et al. Ranolazine reduces Ca2+ overload and oxidative stress and improves mitochondrial integrity to protect against ischemia reperfusion injury in isolated hearts. Pharmacol Res. 2011 Oct;64(4):381-92.

7. Joshua D. Lovelock, Michelle M.et al. Ranolazine Improves Cardiac Diastolic Dysfunction 
Through Modulation of Myofilament Calcium Sensitivity Circ Res. 2012;110:841-850.

8. Flemming Nielsen, Bo Borg Mikkelsen, Jesper Bo Nielsen,et al. Plasma malondialdehyde as biomarker for oxidative stress: reference interval and effects of life-style factors Clinical Chemistry 43:7 1209-1214 (1997)

9. Hale SL Leeka JL, Kloner RA. Improved left ventricular function and reduced necrosis after myocardial ischemia/reperfusion in rabbits treated with ranolazine, an inhibitor of the late sodium channel. J Pharmacol Exp Ther. 2006;318:418-423.

10. Stone P. Ranolazine: New paradigm for management of myocardial ischemia, myocardial dysfunction, and arrhythmias. Cardiol Clin. 2008;26:603-614. 